\title{
Bacteraemia and antibiotic-resistant pathogens in community acquired pneumonia: risk and prognosis
}

\author{
Antoni Torres ${ }^{1}$, Catia Cillóniz ${ }^{1}$, Miquel Ferrer ${ }^{1}$, Albert Gabarrús ${ }^{1}$, \\ Eva Polverino ${ }^{1}$, Santiago Villegas ${ }^{2}$, Francesc Marco ${ }^{3}$, Josep Mensa ${ }^{4}$, \\ Rosario Menéndez ${ }^{5}$ and Michael Niederman ${ }^{6}$
}

\begin{abstract}
Affiliations: ${ }^{1}$ Dept of Pneumology, Institut Clinic del Tórax, Hospital Clinic of Barcelona - Institut d'Investigacions Biomèdiques August Pi i Sunyer (IDIBAPS), University of Barcelona (UB) - SGR 911- Ciber de Enfermedades Respiratorias (Ciberes), Barcelona, Spain. ${ }^{2}$ Dept de Medicina Crítica y Cuidados Intensivos, Universidad CES, Medellin, Colombia. ${ }^{3}$ Microbiology Laboratory (Centre Diagnòstic Biomèdic), Barcelona Centre for International Health Research, Hospital Clínic, Barcelona, Spain. ${ }^{4}$ Dept of Infectious Disease, Hospital Clinic of Barcelona, Barcelona, Spain. ${ }^{5}$ Dept of Pneumology, Hospital La Fe de Valencia, Valencia, Spain. ${ }^{6}$ Dept of Medicine, Winthrop-University Hospital, Mineola, NY, USA.
\end{abstract}

Correspondence: Antoni Torres, Dept of Pneumology, Hospital Clinic of Barcelona, Spain.

E-mail: atorresdaclinic.ub.es

ABSTRACT The sensitivity of blood cultures in the diagnosis of bacteraemia for community-acquired pneumonia is low. Recommendations, by guidelines, to perform blood cultures are discordant. We aimed to determine the incidence, microbial aetiology, risk factors and outcomes of bacteraemic patients with community-acquired pneumonia, including cases with antibiotic-resistant pathogens (ARP).

A prospective, observational study was undertaken on consecutive adult patients admitted to the Hospital Clinic of Barcelona (Barcelona, Spain) with community-acquired pneumonia and blood cultures were obtained.

Of the 2892 patients included, bacteraemia was present in 297 (10\%) patients; 30 (10\%) of whom had ARP (multidrug-resistant Streptococcus pneumoniae, methicillin-resistant Staphylococcus aureus, Pseudomonas aeruginosa, and an extended spectrum of beta-lactamase producing Enterobacteriaceae). In multivariate analyses, pleuritic pain, C-reactive protein $\geqslant 21.6 \mathrm{mg} \cdot \mathrm{dL}^{-1}$ and intensive care unit admissions were independently associated with bacteraemia, while prior antibiotic treatment and pneumococcal vaccine were protective factors. The risk factors for ARP bacteraemia were previous antibiotics and C-reactive protein $<22.2 \mathrm{mg} \cdot \mathrm{dL}^{-1}$, while pleuritic pain was the only protective factor in the multivariate analysis. Bacteraemia (excluding ARP), appropriate empiric treatment, neurological disease, arterial oxygen tension/inspiratory oxygen fraction <250, pneumonia severity index risk classes IV and $\mathrm{V}$, and intensive care unit admission were independently associated with a 30-day hospital mortality in the multivariate analysis. Inappropriate therapy was more frequent in ARP bacteraemia, compared with other bacteraemias ( $27 \%$ versus $3 \%$, respectively, $\mathrm{p}<0.001$ ).

Antibiotic therapy protected against bacteraemia, but increased specifically the risk of bacteraemia from ARP due to the inappropriate coverage of these pathogens. Identifying patients at risk of ARP bacteraemia would help in deciding appropriate empiric antimicrobial therapy. The results from this study provide evidence concerning community-acquired pneumonia patients in whom blood cultures should not be performed.

@ERSpublications

Understand risk factors for ARP bacteraemia and the importance of identifying patients who may have these organisms http://ow.ly/EUs8d

This article has supplementary material available from erj.ersjournals.com

Received: Aug 202014 | Accepted after revision: Nov 192014 | First published online: Jan 222015

Copyright @ERS 2015 


\section{Introduction}

Community-acquired pneumonia (CAP) is a disease with high morbidity and mortality, with short-term mortality reaching $14 \%$ and long-term mortality reaching $50 \%$ within 5 years [1]. CAP with bloodstream infection, continues to be a severe and often life-threatening infection being Streptococcus pneumoniae the most common pathogen, and the leading cause of death $[2,3]$. The association between bacteraemia and mortality is controversial and has been studied mainly for $S$. pneumoniae. Recent studies did not consistently find an association between pneumococcal bacteraemia and mortality [4]. The reason may be that other factors, such as time to the first dose of antibiotics and discordant therapy, are more important determinants of prognosis [5]. The association between mortality and bacteraemic CAP caused by microorganisms other than $S$. pneumoniae is less well-studied.

While the diagnosis of bacteraemia requires blood cultures, the sensitivity of this test in CAP is low $(<20 \%)$. Recommendations of guidelines to perform blood cultures are discordant. While the last Infectious Diseases Society of America (IDSA)/American Thoracic Society (ATS) recommendations limit blood cultures to intensive care unit (ICU) patients [6], the most recent European Respiratory Society/ European Society of Clinical Microbiology and Infectious Diseases guidelines recommend that they should be performed in all hospitalised CAP patients [7]. METERSKy et al. [8] and Falguera et al. [9] described a set of factors (liver disease, pleuritic pain, tachycardia, tachypnea, systolic hypotension, and absence of prior antibiotic treatment [9], liver disease, recent antibiotic treatment, temperature $<35^{\circ} \mathrm{C}$ or $\geqslant 40^{\circ} \mathrm{C}$, blood urea nitrogen $\geqslant 30 \mathrm{mg} \cdot \mathrm{dL}^{-1}$, sodium $<130 \mathrm{mmol} \cdot \mathrm{L}^{-1}$ and white blood cells (WBC) $<5000 \mathrm{~mm}^{-3}$ or $>20000 \mathrm{~mm}^{-3}$ [8]) potentially useful for clinicians to increase the sensitivity of blood cultures and to decrease costs. To our knowledge these criteria have not been applied in clinical practice. In addition the risk factors for bacteraemia caused by "nonstandard-therapy pathogens" have not been described.

We hypothesised that there are specific populations with a lower or higher risk for bacteraemic CAP and that mortality, attributable to bacteraemia, is different according to the type of microorganism isolated. Our goal was to identify risk factors for bacteraemia so that blood culture sampling could focus on this population. An additional aim was to identify bacteraemia caused by antibiotic-resistant pathogens (ARP) and to determine how risk factors for bacteraemia with these organisms differed from risk factors for all bacteraemias.

\section{Materials and methods}

\section{Study design and patients}

Prospective observational study carried out at the Hospital Clinic of Barcelona (Barcelona, Spain), including all adult patients admitted with CAP (from January 2000 to January 2012). Pneumonia was defined as the presence of a new infiltrate on a chest radiograph, together with clinical symptoms suggestive of lower respiratory tract infection. Patients coming from nursing home institutions were included. The exclusion criteria, clinical definitions are described in detail in the supplementary material.

\section{Data collection}

The following parameters were recorded at admission: age, sex, current smoking, alcohol habits, and drug consumption, comorbidities, antibiotic treatment in the 30 days prior to hospital admission, treatment with corticosteroids, clinical symptoms and features, clinical signs, arterial blood gas measurements, chest radiograph findings, laboratory parameters, diagnostic procedures, empiric antibiotic therapy, ventilator support, pulmonary complications, and other clinical events. The duration of treatment, length of hospital stay, and 30-day in-hospital mortality were noted. We also calculated the pneumonia severity index (PSI) and CURB-65 score (confusion, urea $>7 \mathrm{mmol} \cdot \mathrm{L}^{-1}$, respiratory rate $\geqslant 30$ breaths $\cdot \mathrm{min}^{-1}$, blood pressure $<90 \mathrm{mmHg}$ (systolic) $\leqslant 60 \mathrm{mmHg}$ (diastolic), age $\geqslant 65$ years) at admission $[10,11]$.

\section{Microbiological evaluation}

Microbiological examination was performed on sputum, urine, two samples of blood and nasopharyngeal swabs. Pleural fluid, tracheobronchial aspirates and bronchoalveolar lavage (BAL) fluid, when available, were collected for Gram and Ziehl-Neelsen stains and for cultures for bacterial, fungal and mycobacterial pathogens.

Sputum and blood samples were obtained for bacterial culture before starting antibiotic therapy in the emergency department. Nasopharyngeal swab for respiratory virus detection was collected, when available, urine samples for S. pneumoniae and Legionella pneumophila antigen detection were obtained within $24 \mathrm{~h}$

Support statement: This work was supported by Ciber de Enfermedades Respiratorias (CibeRes CB06/06/0028). 2009 Support to Research Groups of Catalonia 911.

Conflict of interest: None declared. 
of hospital admission. Blood samples for serology of atypical pathogens and respiratory viruses were collected at admission and between the third and sixth week thereafter (supplementary material)

\section{Definitions}

Bacteraemic pneumonia was defined as at least one positive blood culture not related to another source of infection or contamination. We considered bacteria contaminants if there was growth of: coagulasenegative Staphylococcus spp., $\alpha$-haemolytic streptococci, Micrococcus spp. or Corynebacterium spp. in one of the two blood cultures.

ARP bacteraemia was defined when it was caused by one of the following microorganisms: methicillin resistant Staphylococcus aureus (MRSA), Enterobactaeriacea producing extended spectrum beta-lactamases and multidrug-resistant (penicillins, beta-lactams, macrolides and quinolones) S. pneumoniae [12]. Pseudomonas aeruginosa cases were also included in this group since it requires a totally different antibiotic treatment.

Appropriateness of empiric antibiotic treatment in all patients was defined according to multidisciplinary guidelines for the management of CAP [13]. Appropriateness of empiric antimicrobial treatment in patients was defined when the isolated pathogens were susceptible in vitro to one or more of the antimicrobials administered. For $P$. aeruginosa infection, appropriate treatment required one active antibiotic against the isolated strain [6].

\section{Statistical analysis}

We show $\mathrm{n}$ (\%) for categorical variables and median (interquartile range) for continuous variables with non-normal distribution or mean $\pm \mathrm{SD}$ ) for those with normal distribution. Categorical variables were compared using the Chi-square test or Fisher's exact test. Continuous variables were compared using the Student t-test or the nonparametric Mann-Whitney U-test. Univariate and multivariate logistic regression analyses were performed to identify variables predictive of patients with bacteraemic CAP and patients ARP bacteraemia, respectively (dependent variables). Also univariate and multivariate logistic regression analyses were performed to predict 30-day hospital mortality (dependent variable); we also performed a subgroup analysis for patients with bacteraemia. Variables that showed a significant result univariately $(p<0.1)$ were included in the corresponding multivariate, logistic regression backward stepwise model (supplementary methods). Variables highly correlated were excluded from multivariate analyses. The Hosmer-Lemeshow goodness-of-fit test was performed to assess the overall fit of the models [14]. Receiver operating characteristic (ROC) curves were constructed for the ability to predict bacteraemia, patients with ARP bacteraemia, and 30-day hospital mortality, using significant variables derived from the respective logistic regression models. The level of significance was set at 0.05 (two-tailed). All analyses were performed with IBM SPSS Statistics 18.0 (Armonk, NY, USA).

\section{Results}

\section{Patients' characteristics}

Of the 3719 patients with CAP admitted during the observation period, 827 (22\%) were excluded for unavailable blood cultures. Clinical characteristics and outcomes comparing patients who received or not

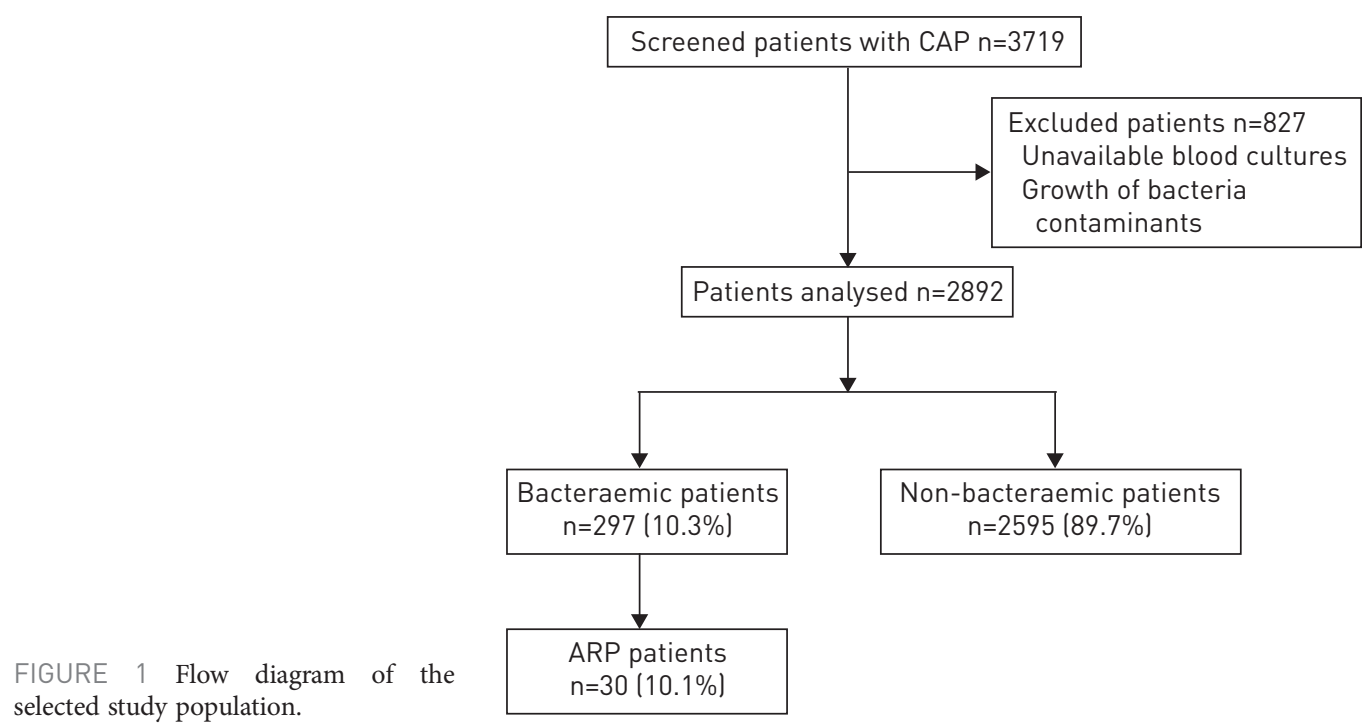


blood culture are described in table S1. The study population therefore comprised a total of 2892 patients evaluated in the emergency deparment with a diagnosis of CAP (fig. 1).

Altogether, 297 (10\%) patients had bacteraemia. On average, patients with bacteraemia were aged <65 years, less frequently males, less often ex-smokers, had less often received prior antibiotics, had a lower rate of pneumococcal vaccination, had a lower incidence of chronic cardiovascular disease, a higher frequency of PSI risk class IV and V, and a CURB-65 risk class 3-5 when compared with non-bacteraemic patients. They also more likely to have creatinine $\geqslant 1.5 \mathrm{mg} \cdot \mathrm{dL}^{-1}$, C-reactive protein $\geqslant 21.6 \mathrm{mg} \cdot \mathrm{dL}^{-1}, \mathrm{WBC}$ counts $\geqslant 10 \times 10^{9} \mathrm{~L}^{-1}$, oxygen saturation levels $<92 \%$ and arterial oxygen tension $\left(\mathrm{PaO}_{2}\right) /$ inspiratory oxygen fraction $\left(\mathrm{FiO}_{2}\right)<250$. Patients with bacteraemia had a significantly longer median length of hospital stay, more frequent admission to the ICU, more need for either invasive or noninvasive mechanical ventilation. On average, patients with bacteraemia were more frequently classified as moderate-to-severe by the Fine score (PSI risk class IV and V). The crude 30-day mortality rate was significantly higher in bacteraemic patients (table 1 and S2).

Of the 297 patients with bacteraemia, $16(5.4 \%)$ were treated as outpatients, 188 (63.3\%) were admitted to a ward and $93(31.3 \%)$ were admitted to the ICU. The distribution of bacteraemic patients by site of care, PSI and CURB-65 scores is shown in figures S1, S2 and S3, respectively.

\section{Microbial aetiology and susceptibility}

Overall aetiology was established in 1292 (45\%) patients (table S3). In bacteraemic patients the main microorganisms isolated were S. pneumoniae $(\mathrm{n}=249,84 \%)$, S. aureus $(\mathrm{n}=16,5 \%)$, Escherichia coli $(\mathrm{n}=9$, $3 \%)$, and Haemophilus influenzae ( $\mathrm{n}=7,2 \%)$. ARP were present in $10 \%$ of cases $(\mathrm{n}=30)$ (table 2$)$.

\section{TABLE 1 Clinical and epidemiological characteristics of bacteraemic and non-bacteraemic} community-acquired pneumonia (CAP) patients

\begin{tabular}{|c|c|c|c|}
\hline & Bacteraemic CAP & Non-bacteraemic CAP & p-value \\
\hline Patients $\mathrm{n}$ & 267 & 2595 & \\
\hline \multicolumn{4}{|l|}{ Demographic } \\
\hline Age $\geqslant 65$ years & $156 \pm 52.5$ & $1535 \pm 59.2$ & 0.028 \\
\hline Male & $167(56.2)$ & $1636(63.0)$ & 0.022 \\
\hline Current smoker & 83 (28.3) & $650(25.1)$ & 0.24 \\
\hline Current alcohol consumer & $48(16.4)$ & $377(14.6)$ & 0.43 \\
\hline Previous antibiotic ${ }^{\#}$ & 35 (12.5) & $560(22.4)$ & $<0.001$ \\
\hline Influenza vaccine & $83(37.4)$ & $958(43.9)$ & 0.062 \\
\hline Pneumococcal vaccine & $22(10.0)$ & $367(16.9)$ & 0.008 \\
\hline Inhaled corticosteroid & $50(17.2)$ & $462(18.0)$ & 0.74 \\
\hline Systemic corticosteroid & $3(1.1)$ & $36(1.5)$ & 0.61 \\
\hline Comorbidities? & 185 (62.3) & $1643(63.3)$ & 0.73 \\
\hline Chronic respiratory disease & $108(36.4)$ & 968 (37.3) & 0.75 \\
\hline Chronic cardiovascular disease & $37(12.5)$ & $459(18.8)$ & 0.024 \\
\hline Diabetes mellitus & $52(18.2)$ & $417(16.6)$ & 0.51 \\
\hline Neurological disease & $52(17.7)$ & $438(17.0)$ & 0.78 \\
\hline Chronic renal disease & $16(5.4)$ & $165(6.4)$ & 0.52 \\
\hline Chronic liver disease & $18(6.1)$ & $107(4.1)$ & 0.12 \\
\hline Nursing-home $\#$ & $56(2.2)$ & 5 (1.7) & 0.59 \\
\hline CURB-65 risk class $3-5$ & $76(25.9)$ & $436(17.6)$ & 0.001 \\
\hline PSI risk class IV and V & $166(55.9)$ & $1261(48.6)$ & 0.017 \\
\hline ICU admission & 93 (31.3) & $445(17.1)$ & $<0.001$ \\
\hline Mechanical ventilation ${ }^{+}$ & & & 0.001 \\
\hline Not ventilated & $255(85.9)$ & $2390(92.1)$ & $<0.001$ \\
\hline Noninvasive & $18(6.1)$ & $92(3.5)$ & 0.032 \\
\hline Invasive & $24(8.1)$ & $113(4.4)$ & 0.004 \\
\hline Length of hospital stay days & $9.0(6.0-13.0)$ & $6.0(4.0-10.0)$ & $<0.001$ \\
\hline 30-day mortality & $33(11.1)$ & $154(5.9)$ & 0.001 \\
\hline Appropriate empiric treatment & $282(94.9)$ & 2446 (95.2) & 0.86 \\
\hline
\end{tabular}

Data are presented as median \pm SD, $\mathrm{n}(\%)$ or median (interquartile range), unless otherwise stated. Percentages calculated on non-missing data. CURB-65: confusion, urea $>7 \mathrm{mmol} \cdot \mathrm{L}^{-1}$, respiratory rate $\geqslant 30$ breaths $\mathrm{min}^{-1}$, blood pressure $<90 \mathrm{mmHg}$ (systolic) $\leqslant 60 \mathrm{mmHg}$ (diastolic), age $\geqslant 65$ years; PSI: pneumonia severity index; ICU: intensive care unit. \#: variables included in the definition of healthcare associated pneumonia; ": patients could have $>1$ comorbidity; ${ }^{+}$: patients who initially received noninvasive ventilation but subsequently needed intubation were included in the invasive mechanical ventilation group. Bold indicates statistically significant 
TABLE 2 Pathogens isolated in blood culture from the 297 patients with bacteraemic community acquired pneumonia

Isolate

\begin{tabular}{|c|c|}
\hline Streptococcus pneumoniae & 249 (83.8) \\
\hline S. pneumoniae multiresistant ${ }^{\#}$ & $5(1.7)$ \\
\hline Staphylococcus aureus & $16(5.4)$ \\
\hline MRSA $^{\#}$ & $14(4.7)$ \\
\hline MSSA & $2(0.7)$ \\
\hline Escherichia coli & $9(3.0)$ \\
\hline E. coli beta-lactamase producers ${ }^{\#}$ & $6(2.0)$ \\
\hline Haemophilus influenzae & $7(2.4)$ \\
\hline Enterobacter sp. & $4(1.3)$ \\
\hline Enterobacter spp. beta-lactamase producers & $1(0.3)$ \\
\hline Klebsiella pneumoniae & $4(1.3)$ \\
\hline K. pneumoniae beta-lactamase producers ${ }^{\#}$ & 2 (0.7) \\
\hline Acinetobacter baumannii & $2(0.7)$ \\
\hline Proteus sp. & $2(0.7)$ \\
\hline Proteus spp. beta-lactamase producers ${ }^{\#}$ & $1(0.3)$ \\
\hline Moraxella catarrhalis & $1(0.3)$ \\
\hline Peptostreptococcus sp. & $1(0.3)$ \\
\hline Pseudomonas aeruginosa" & $1(0.3)$ \\
\hline Streptococcus pyogenes & $1(0.3)$ \\
\hline
\end{tabular}

Data are presented as n (\%). MRSA: methicillin-resistant S. aureus; MSSA: methicillin-sensitive S. aureus.

\#: antibiotic resistant pathogens $(\mathrm{n}=30)$.

12 cases (40\%) of ARP bacteraemia were admitted to ICU. Complete susceptibility data are described in detail in the supplementary results.

Empiric antibiotic therapy

Initial empiric antibiotic treatment was inappropriate in 15 (5\%) of 297 patients with bacteraemic CAP and five patients with inappropriate treatment died (33\%). Inappropriate therapy was given to eight of the 30 patients $(27 \%)$ with ARP bacteraemia versus 7 of the 267 with other bacteraemias $(3 \%)(\mathrm{p}<0.001)$. Data of complete empiric antibiotic therapy are described in detail in the supplementary results. The pathogens

TABLE 3 Significant univariate and multivariate logistic regression analyses of predictors for bacteraemic community acquired pneumonia

\begin{tabular}{|c|c|c|c|c|}
\hline & \multicolumn{2}{|c|}{ Univariate } & \multicolumn{2}{|c|}{ Multivariate } \\
\hline & OR $(95 \% \mathrm{CI})$ & p-value & OR $(95 \% \mathrm{CI})$ & p-value \\
\hline Pneumococcal vaccine & $0.55(0.35-0.86)$ & 0.009 & $0.59(0.35-0.99)$ & 0.047 \\
\hline Influenza vaccine & $0.76(0.57-1.01)$ & 0.062 & & - \\
\hline Previous antibiotic & $0.49(0.34-0.71)$ & $<0.001$ & $0.51(0.32-0.79)$ & 0.003 \\
\hline Pleuritic pain & $2.20(1.71-2.83)$ & $<0.001$ & $2.21(1.60-3.06)$ & $<0.001$ \\
\hline C-reactive protein $\geqslant 21.6 \mathrm{mg} \cdot \mathrm{dL}^{-1}$ & $2.65(2.01-3.50)$ & $<0.001$ & $2.36(1.70-3.27)$ & $<0.001$ \\
\hline White blood cell count $\geqslant 10 \times 10^{9} \cdot \mathrm{L}^{-1}$ & $1.41(1.07-1.85)$ & 0.015 & & - \\
\hline PSI risk class IV and V & $1.34(1.05-1.71)$ & 0.017 & & - \\
\hline CURB-65 risk class 3-5 & $1.64(1.24-2.17)$ & 0.001 & & - \\
\hline ICU admission & $2.20(1.69-2.87)$ & $<0.001$ & $1.80(1.27-2.56)$ & 0.001 \\
\hline Mechanical ventilation" & & 0.002 & & \\
\hline Not ventilated & 1 & & & \\
\hline Noninvasive & $1.83(1.09-3.09)$ & 0.023 & & \\
\hline Invasive & $1.99(1.26-3.15)$ & 0.003 & & \\
\hline
\end{tabular}

PSI: pneumonia severity index; CURB-65: confusion, urea $>7 \mathrm{mmol} \cdot \mathrm{L}^{-1}$, respiratory rate $\geqslant 30$ breaths $\cdot \mathrm{min}^{-1}$, blood pressure $<90 \mathrm{mmHg}$ (systolic) $\leqslant 60 \mathrm{mmHg}$ (diastolic), age $\geqslant 65$ years; ICU: intensive care unit; \#: Hosmer-Lemeshow goodness-of-fit test, $p=0.32$; ${ }^{\text {ๆ }}$ : the $p$-value corresponds to differences between the three groups (not ventilated, non-invasive or invasive). 
TABLE 4 Probability of bacteraemic CAP

Risk factors

Protective factors

\begin{tabular}{cccc}
$\begin{array}{c}\text { Previous antibiotic } \\
\text { and pneumococcal } \\
\text { vaccine }\end{array}$ & $\begin{array}{c}\text { Previous } \\
\text { antibiotic }\end{array}$ & $\begin{array}{c}\text { Pneumococcal } \\
\text { vaccine }\end{array}$ & None \\
\hline 1.3 & 2.2 & 2.5 & 4.3 \\
2.3 & 3.9 & 4.5 & 7.4 \\
5.0 & 8.2 & 9.4 & 15.1 \\
5.3 & 8.7 & 10.0 & 15.9 \\
11.1 & 17.5 & 19.7 & 29.5 \\
& & & \\
2.8 & 4.8 & 5.5 & 9.0 \\
6.5 & 10.5 & 12.0 & 18.9 \\
3.0 & 5.0 & 5.8 & 9.5 \\
\hline
\end{tabular}

Data are presented as \%. ICU: intensive care unit.

associated with inappropriate treatment were E. coli $(\mathrm{n}=4), S$. aureus $(\mathrm{n}=4)$, H. influenzae $(\mathrm{n}=2)$, Klebsiella pneumoniae $(\mathrm{n}=2)$, S. pneumoniae $(\mathrm{n}=1)$, Proteus sp. $(\mathrm{n}=1)$ case and Acinetobacter $(\mathrm{n}=1)$ case (table S4).

\section{Predictors of bacteraemic CAP}

Several variables were significantly associated with bacteraemic CAP in the univariate logistic regression analyses (table 3). Among these variables, pleuritic pain, C-reactive protein $\geqslant 21.6 \mathrm{mg} \cdot \mathrm{dL}^{-1}$ and ICU admission were risk factors in the multivariate analysis. Alternatively, prior antibiotic treatment and pneumococcal vaccine were protective against the development of bacteraemia in the multivariate analysis. The area under the ROC curve was 0.71 (95\% CI 0.68-0.75) for the model predictive of bacteraemia (fig. S4). Predictors from the model were used to calculate the probability of bacteraemia by the following formula. $\operatorname{Exp}(\beta) /(1+\operatorname{Exp}(\beta))$, where $\beta=-3.111-0.682$ (in case of prior antibiotic treatment) -0.533 (in case of pneumococcal vaccine) +0.588 (in case of ICU admission) +0.795 (in case of pleuritic pain) +0.858 (if C-reactive protein $\geqslant 21.6 \mathrm{mg} \cdot \mathrm{dL}^{-1}$ ). Using this model, the probability of bacteraemia for patients without any of these risk factors and with the two protective factors was $1 \%$, being $30 \%$ for patients showing all three risk factors and without any of the protective factors.

The probabilities of bacteraemia for the different combinations of risk and protective factors are shown in tables 4 and 5, and demonstrate that the risk of bacteraemia could be predicted by assessing the number of clinical predictors present. We performed the same analysis excluding cases of ARP bacteraemia and the independent predictors for bacteraemia were the same.

\section{Predictors of patients with ARP bacteraemia}

In the subgroup of bacteraemic patients, variables significantly associated with patients having ARP bacteraemia in the univariate logistic regression analyses were previous antibiotic treatment, neurological disease, being in a nursing home, pleuritic pain, C-reactive protein, and CURB-65. Among these variables,

TABLE 5 Recommendation for not performing blood cultures in immunocompetent patients with community-acquired pneumonia (CAP)

\section{Previous antibiotic treatment \\ Previous pneumococcal vaccination \\ No intensive care unit admission \\ Absence of pleuritic pain \\ C-reactive protein $<21.6 \mathrm{mg} \cdot \mathrm{dL}^{-1}$}

The risk of having bacteraemia with one, two, three, four or five factors is $19 \%, 11 \%, 3 \%, 2 \%$ and $2 \%$, respectively. The risk of having bacteraemia (after excluding previous pneumococcal vaccination) with one, two, three or four factors is $19 \%, 10 \%, 4 \%$ and $3 \%$, respectively. Previous antibiotic treatment refers to if patients received antibiotics any time during the 2 weeks prior to hospital admission for CAP. Pneumococcal vaccination refers to the 23-valent polysaccharide pneumococcal vaccine (PPV23), if the patient received a vaccine in the last 5 years. Serotypes covered by PPV23 have shifted and now the indicated vaccine is the conjugated 13 valent. 
TABLE 6 Significant univariate and multivariate logistic regression analyses for the prediction of patients with antibiotic-resistant pathogen bacteraemia

\begin{tabular}{|c|c|c|c|c|}
\hline & \multicolumn{2}{|c|}{ Univariate } & \multicolumn{2}{|c|}{ Multivariate $^{\#}$} \\
\hline & OR $(95 \% \mathrm{CI})$ & p-value & OR $(95 \% \mathrm{CI})$ & p-value \\
\hline Previous antibiotic & $2.55(1.00-6.50)$ & 0.051 & $3.70(1.24-11.03)$ & 0.019 \\
\hline Neurological disease & $2.20(0.94-5.14)$ & 0.067 & & \\
\hline Nursing-home ${ }^{\text {I }}$ & $6.21(1.00-38.78)$ & 0.051 & & \\
\hline Pleuritic pain & $0.39(0.18-0.87)$ & 0.022 & $0.24(0.09-0.63)$ & 0.004 \\
\hline C-reactive protein $<22.2 \mathrm{mg} \cdot \mathrm{dL}^{-1}$ & $4.64(1.86-11.61)$ & 0.001 & $4.09(1.55-10.78)$ & 0.004 \\
\hline CURB-65 risk class 3-5 & $2.07(0.95-4.53)$ & 0.068 & & \\
\hline
\end{tabular}

CURB-65: confusion, urea $>7 \mathrm{mmol} \cdot \mathrm{L}^{-1}$, respiratory rate $\geqslant 30$ breaths $\cdot \mathrm{min}^{-1}$, blood pressure $<90 \mathrm{mmHg}$ (systolic) $\leqslant 60 \mathrm{mmHg}$ (diastolic), age $\geqslant 65$ years. \#: Hosmer-Lemeshow goodness-of-fit test, $p=0.70$; ๆ: variables included in the definition of healthcare associated pneumonia.

previous antibiotic and C-reactive protein $<22.2 \mathrm{mg} \cdot \mathrm{dL}^{-1}$ were risk factors in the multivariate analysis, while pleuritic pain was the only protective factor (table 6).

The area under the ROC curve was 0.76 (95\% CI 0.66-0.87) for the model predictive of patients with ARP bacteraemia (fig. 2). Predictors from the model were used to calculate the probability of patients with ARP bacteraemia given by the following formula: $\operatorname{Exp}(\beta) /(1+\operatorname{Exp}(\beta))$, where $\beta=-2.477+1.307$ (in case of prior antibiotic treatment) -1.428 (in case of pleuritic pain) +1.408 (if C-reactive protein $<22.2 \mathrm{mg} \cdot \mathrm{dL}^{-1}$ ). Using this model, the probability for ARP bacteraemia for patients without any of these risk factors and with the protective factor was $2 \%$, being $47 \%$ for patients showing the two risk factors and without the protective factor.

In addition we performed a multivariate analysis comparing the 30 cases of ARP bacteraemia versus total population only CURB-65 risk class 3-5 (OR 2.46, 95\% CI 1.15-5.27, p=0.020) and ICU admission (OR $1.99,95 \%$ CI $1.13-5.19, \mathrm{p}=0.022)$ resulted as significant in this analysis. The probability of ARP bacteraemia in patients admitted to the ICU and CURB-65 risk class 3-5 was 3.8\%).

\section{Predictors of 30-day hospital mortality}

Table 7 shows the crude 30-day hospital mortality in relation to the different microorganisms. The crude mortality of $S$. pneumoniae bacteraemia was $9 \%$, while the mortality of the ARP bacteraemias was at least two-fold higher (23\%; S. aureus five out of 14 cases, E. coli one out of six cases and K. pneumoniae one of two cases).

FIGURE 2 Receiver operating characteristic analysis of significant variables derived from the logistic regression model in their capacity to predict patients with bacteraemia caused by antibiotic-resistant pathogens. AUC: area under the curve.

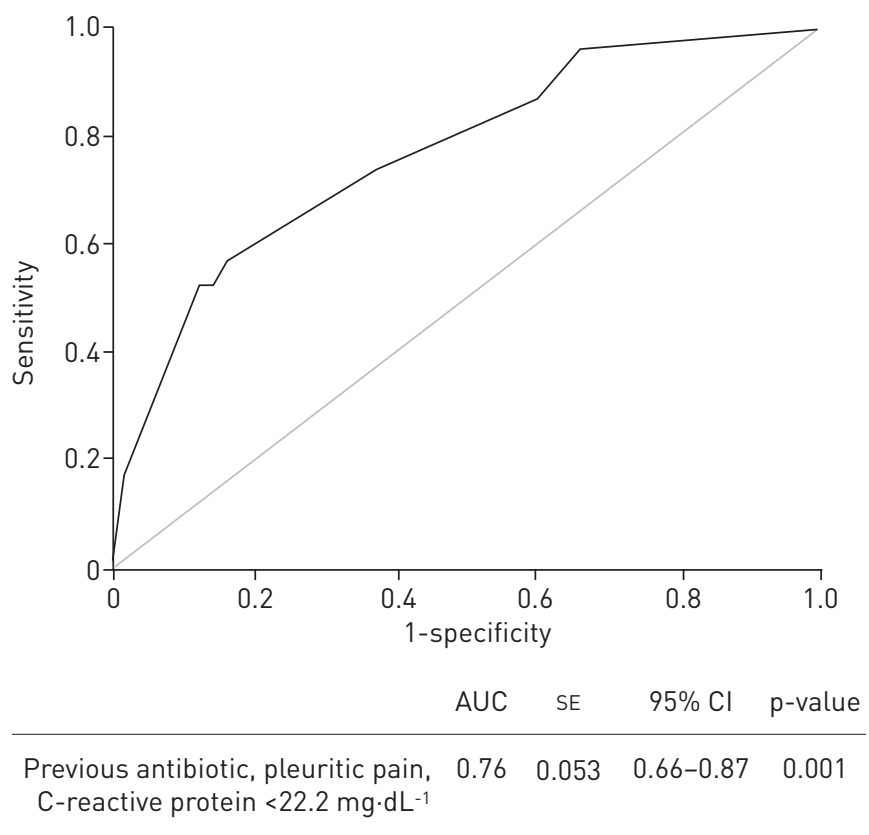


TABLE 7 30-day hospital mortality according to microbial aetiology

\begin{tabular}{|c|c|}
\hline Streptococcus pneumoniae & $23 / 249(9.2)$ \\
\hline Streptococcus pneumoniae multiresistant ${ }^{\#}$ & $0 / 5(0)$ \\
\hline Staphylococcus aureus & $6 / 16(37.5)$ \\
\hline MRSA $^{\#}$ & $5 / 14(35.7)$ \\
\hline MSSA & $1 / 2(50.0)$ \\
\hline Escherichia coli & $2 / 9(22.2)$ \\
\hline Escherichia coli BL producers ${ }^{\#}$ & $1 / 6(16.7)$ \\
\hline Haemophilus influenzae & $1 / 7(14.3)$ \\
\hline Enterobacter sp. & $0 / 4(0)$ \\
\hline Enterobacter sp. beta-lactamase producers ${ }^{\#}$ & $0 / 1(0)$ \\
\hline Klebsiella pneumonia & $1 / 4(25.0)$ \\
\hline Klebsiella pneumoniae beta-lactamase producers $\#$ & $1 / 2(50.0)$ \\
\hline Acinetobacter baumannii & $0 / 2(0)$ \\
\hline Proteus sp. & $0 / 2(0)$ \\
\hline Proteus spp. beta-lactamase producers $\#$ & $0 / 1(0)$ \\
\hline Moraxella catarrhalis & $0 / 1(0)$ \\
\hline Peptostreptococcus sp. & $0 / 1(0)$ \\
\hline Pseudomonas aeruginosa" & $0 / 1(0)$ \\
\hline Streptococcus pyogenes & $0 / 1(0)$ \\
\hline Total & $33 / 297(11.1)$ \\
\hline
\end{tabular}

Data are presented as $n / N(\%)$, where $n=$ number of patients who died and $N=$ total number of patients. MRSA: methicillin-resistant S. aureus; MSSA: methicillin-sensitive S. aureus. \#: antibiotic-resistant pathogen bacteraemia $(7 / 30(23.3 \%))$.

The percentage of patients with bacteraemia was higher in non-survivors when compared with survivors $(\mathrm{n}=33(18 \%)$ versus $\mathrm{n}=264$ (10\%), respectively, $\mathrm{p}=0.001)$. In particular ARP bacteraemia was higher in non-survivors when compared with survivors $(n=7$ (4\%) versus $n=23(1 \%), p<0.001)$.

In the multivariate logistic regression analysis (table 8), bacteraemia (excluding ARP), neurological disease, $\mathrm{PaO}_{2} / F_{\mathrm{IO}}<250$, PSI risk classes IV and $\mathrm{V}$, and ICU admission were risk factors for 30-day hospital mortality, while appropriate empiric treatment was the only protective factor. The area under the ROC curve was 0.80 (95\% CI 0.76-0.83) (fig. S5).

In the subgroup of bacteraemic patients, several variables were significantly associated with 30-day hospital mortality in the univariate logistic regression analyses (table S5). The multivariate analysis showed that dyspnoea, PSI risk classes IV and V and ICU admission were risk factors for 30-day hospital mortality while cough and appropriate empiric treatment were protective factors The area under the ROC curve was 0.84 (95\% CI 0.78-0.90) for the model predictive of 30-day hospital mortality (fig. S6).

\section{Discussion}

The main findings of this study can be summarised as follows. 1) The prevalence of bacteraemia in this large series of consecutive patients with CAP was $10 \%$, of which $5 \%$ were not admitted to the hospital. 2) Almost $20 \%$ of the cases were due to microorganisms other than S. pneumonia. 3) Of particular interest, $10 \%$ of bacteraemic cases were caused by ARP. 4) We identified several risk factors to predict bacteraemia in general and ARP bacteraemia, and prior antibiotic therapy was protective against bacteraemia in general but a risk factor for ARP bacteraemia. 5) Bacteraemia (excluding ARP) was a risk factor associated with CAP mortality, while appropriate empiric treatment was a protective factor in a multivariate model. ARP bacteraemia was associated with a significantly higher rate of inappropriate therapy than bacteraemia with other pathogens (27\% versus $2.6 \%$ ).

The diagnostic yield of blood cultures was $10 \%$ in this series. This prevalence is in the low range of the results reported in the literature $[9,15]$. The prevalence of bacteraemia increased according to severity of illness (defined by PSI and CURB-65 scores), and in relation to site of care, with the highest rate being in ICU admitted patients (31\%) and only $5 \%$ in patients not admitted to the hospital. The factors that may affect the positivity of blood cultures are prior antibiotic treatment, timing to blood culture sampling and accurate microbiological processing $[16,17]$. In this study we could only assess the first factor and we found that without previous antibiotic treatment, the prevalence was almost doubled. This was also confirmed when analysing patients according to the probability of bacteraemia (table 4). However, if bacteraemia was present in spite of prior antibiotic therapy, it was more likely to be with ARP. METERSKY et al. [8] reported a large retrospective series of CAP patients with bacteraemia, they found that liver disease, vital sign 
TABLE 8 Significant univariate and multivariate logistic regression analyses for the prediction of 30-day hospital mortality in the total population

\begin{tabular}{|c|c|c|c|c|}
\hline & \multicolumn{2}{|c|}{ Univariate } & \multicolumn{2}{|c|}{ Multivariate $^{\#}$} \\
\hline & OR $(95 \% \mathrm{CI})$ & p-value & OR $(95 \% \mathrm{CI})$ & p-value \\
\hline Influenza vaccine & $1.76(1.20-2.59)$ & 0.004 & & \\
\hline Diabetes mellitus & $1.49(1.03-2.15)$ & 0.034 & & \\
\hline Neurological disease & $3.48(2.54-4.77)$ & $<0.001$ & $1.99(1.33-3.00)$ & 0.001 \\
\hline Cough & $0.61(0.44-0.86)$ & 0.005 & & \\
\hline Dyspnoea & $3.07(2.04-4.63)$ & $<0.001$ & & \\
\hline Pleuritic pain & $0.45(0.32-0.64)$ & $<0.001$ & & \\
\hline $\mathrm{PaO}_{2} / \mathrm{FiO}_{2}<250$ & $5.07(3.54-7.26)$ & $<0.001$ & $3.21(2.19-4.71)$ & $<0.001$ \\
\hline Nursing-home & $5.10(2.79-9.32)$ & $<0.001$ & & \\
\hline PSI risk class IV and V & $7.74(5.01-11.91)$ & $<0.001$ & $4.13(2.46-6.95)$ & $<0.001$ \\
\hline CURB-65 risk class 3-5 & $5.45(3.98-7.46)$ & $<0.001$ & & \\
\hline ICU admission & $3.16(2.32-4.31)$ & $<0.001$ & $1.85(1.26-2.73)$ & 0.002 \\
\hline Mechanical ventilation" & & $<0.001$ & & \\
\hline Not ventilated & 1 & & & \\
\hline Noninvasive & $3.69(2.13-6.37)$ & $<0.001$ & & \\
\hline Invasive & $9.86(6.62-14.70)$ & $<0.001$ & & \\
\hline Length of hospital stay ${ }^{+}>7$ days & $1.19(1.11-1.29)$ & $<0.001$ & & \\
\hline Appropriate empiric treatment & $0.29(0.18-0.44)$ & $<0.001$ & $0.28(0.16-0.50)$ & $<0.001$ \\
\hline Bacteraemia $\$$ & & $<0.001$ & & 0.011 \\
\hline Non-bacteraemic CAP & 1 & & & \\
\hline Non-ARP bacteraemia & $1.71(1.11-2.64)$ & 0.016 & $1.94(1.18-3.19)$ & 0.009 \\
\hline ARP bacteraemia & $4.82(2.04-11.42)$ & $<0.001$ & $2.48(0.82-7.54)$ & 0.11 \\
\hline
\end{tabular}

$\mathrm{PaO}_{2}$ : arterial oxygen tension; $\mathrm{FlO}_{2}$ : inspiratory oxygen fraction; PSI: pneumonia severity index; CURB-65: confusion, urea $>7 \mathrm{mmol} \cdot \mathrm{L}^{-1}$, respiratory rate $\geqslant 30$ breaths $\cdot \mathrm{min}^{-1}$, blood pressure $<90 \mathrm{mmHg}$ (systolic) $\leqslant 60 \mathrm{mmHg}$ (diastolic), age $\geqslant 65$ years; ICU: intensive care unit; CAP: community acquired pneumonia; ARP: antibiotic-resistant pathogens. \#: Hosmer-Lemeshow goodness-of-fit test, $p=0.050$; : the $p$-value corresponds to differences between the three groups (not ventilated, noninvasive and invasive); ${ }^{+}$: length of hospital stay was treated as a continuous variable and $>7$ days indicates the increase by 7 days; ${ }^{\S}$ : the p-value corresponds to the differences between the three groups (non-bacteraemic CAP, non-ARP bacteraemia or ARP bacteraemial.

abnormalities, blood urea nitrogen elevation, sodium abnormalities and WBC elevation were risk factors for bacteraemia in both a derivation and validation cohort. They proposed a score with two categories of risk, low and high. They did not stratify these risks in relation to specific microorganisms.

FALGUERA et al. [9] did a similar analysis using a database of prospectively collected data. Chronic liver disease, pleuritic pain and vital sign abnormalities were the predictors of bacteraemia. They also proposed a score, giving one point to each one of the factors and recommended obtaining blood cultures in patients with a score $>2$. The area under the curve (AUC) of this score was 0.70 , but was not adjusted for the different microorganisms. In both studies prior antibiotics were a protective factor [8, 9]. To our knowledge neither of these two scores have been implemented in clinical practice.

In our study we found several risk factors for bacteraemia using multivariate logistic regression analyses, including pleuritic pain, C-reactive protein $\geqslant 21.6 \mathrm{mg} \mathrm{dL}^{-1}$ and ICU admission. The AUC for the model was 0.71 (95\% CI 0.68-0.75). Interestingly, we found that in the presence of all three risk factors and the absence of the two protective factors, the probability of bacteraemia was $30 \%$. This rule could help to define which patients would benefit from having blood cultures taken and more intensive monitoring [1] and those who would not. Currently, only the last IDSA/ATS [6] guidelines have tried to define which population of CAP patients would benefit from blood cultures, while other guidelines recommend blood samples for all hospitalised patients with CAP [7]. New CAP guidelines should focus on which patients should not undergo blood cultures and our results could help with this specific aspect. By contrast to Metersky et al. [8] and Falguera et al. [9] we did not find that chronic liver disease was a risk factor for bacteraemia. This can be explained by the fact that not all our patients had liver cirrhosis, which is probably the real risk factor for bacteraemia.

Our results concerning ARP bacteraemia are of interest in the context of an increasing incidence of resistant microorganisms in the community. In our study this problem was of low magnitude since only $30(10 \%)$ patients of the total bacteraemic CAP cases had ARP. In our definition of ARP we included 
those microorganisms that cannot be treated with the usual empiric therapy for CAP (MRSA, multidrug-resistant S. pneumoniae, $P$. aeruginosa, extended-spectrum beta-lactamase producing (ESBL) Enterobacteriacea. We recognise that $P$. aeruginosa is not purely an ARP microorganism but we felt that it would be better to include it in this group since it requires a totally different antibiotic treatment. This is an important group to identify, because these patients had a high frequency of inappropriate empiric therapy, and possibly, by using the risk factor profile that we identified, these patients could be anticipated and treated with appropriate empiric therapy more often. In our study, prior antibiotic treatment, C-reactive protein blood levels and pleuritic pain (protective) were the main predictors of these microorganisms. Nursing homes were not a risk factor for ARP bacteraemia in the multivariate analysis and this can be explained by the heterogeneity of nursing home patients in Spain, which include patients with no risk. The combination of these three factors could be useful to suspect ARP bacteraemia in CAP; the AUC for the model was 0.77 (95\% CI 0.66-0.87). In a second multivariate analysis (ARP bacteremic versus all population) we found that CURB-65 risk class 3-5 and ICU admission were the only two factors associated with a higher risk of ARP bacteraemia, suggesting that the problem of ARP bacteraemia is restricted to more severe patients.

Interestingly we included in our ARP group multiresistant S. pneumonia, since these microorganisms cannot be treated with the standard recommended antibiotics. Recent publications from SHORR et al. [18] and AliberTi et al. [19] have defined risk factors for these types of microorganisms in CAP patients, but not specifically in patients with bacteraemia. In addition they did not include multidrug-resistant S. pneumoniae. Aliberti et al. [20], in a validation study, found that the score from Shorr et al. [18] and the score that they proposed had an AUC of 0.79 and 0.71 respectively. We found several factors associated with 30-day hospital mortality in the overall population: bacteraemia, inappropriate empiric treatment, neurological disease, $\mathrm{PaO}_{2} / \mathrm{FIO}_{2}<250$, PSI risk classes IV and V and ICU admission. Bacteraemia (excluding ARP) was an independent risk factor for mortality, which reinforces the importance of the suspicion and early detection of these patients. However, in multivariate analysis we could not find an independent association between ARP bacteraemia and mortality. This is probably due to the small number of ARP bacteraemia. Alternatively, our data suggest that early and appropriate antibiotic treatment is a key factor for mortality and, in fact, we did find a significantly higher rate of inappropriate empiric therapy with ARP bacteraemia than with other types of bacteraemic CAP (27\% versus $2.6 \%$ ).

The major strength of this study is that we provide original data on the burden of ARP bacteraemia in CAP, and define the risk factors associated with these organisms. In addition, and in contrast with previous studies, notably METERSKY [8] and FALGUERA [9], we performed analyses of risk factors and mortality for the group as a whole, as well as for ARP. We also calculated the probability of bacteraemia in the presence or absence of risk factors for each of these two populations. We also provide indications of which population of CAP would not potentially benefit from undergoing blood cultures.

Our study has limitations. First, this is a very specific Spanish population and resistance patterns in our country are different from those in other countries, consequently the findings of our study need to be confirmed in a validation cohort outside of Spain. Second, we did not record the time to the first dose of antibiotic, which is a factor that has been associated with mortality in the most severe CAP patients. Third, a potential limitation is the long period of the study. However, our protocols and microbiological procedures have not substantially changed during these years. Fourth, blood cultures were not performed on all patients and, therefore, could be a potential bias that needs to be taken into consideration.

Finally we do not have data concerning all antibiotic changes that were undertaken once the blood culture results were known. However, in 17 out 30 ARP bacteraemia, initial antibiotic treatment was inadequate and antibiotics were modified according the corresponding antibiogram.

In summary, we have described clinical predictors of bacteraemic CAP and ARP. Importantly, we provide information about CAP patients in whom blood cultures should not be performed (table 5). Mortality in our CAP population was significantly associated with bacteraemia and treatment inadequacy, which was more common with ARP bacteraemia than with other forms of bacteraemia.

\section{Acknowledgements}

We are indebted to all medical and nursing colleagues for their assistance and cooperation in this study.

\section{References}

1 Ewig S, Torres A. Community-acquired pneumonia as an emergency: time for an aggressive intervention to lower mortality. Eur Respir J 2011; 38: 253-260.

2 Niederman MS, Mandell LA, Anzueto A, et al. Guidelines for the management of adults with communityacquired pneumonia: diagnosis, assessment of severity, antimicrobial therapy, and prevention. Am J Respir Crit Care Med 2001; 163: 1730-1754. 
3 Lin SH, Lai CC, Tan CK, et al. Outcomes of hospitalized patients with bacteraemic and non-bacteraemic community-acquired pneumonia caused by Streptococcus pneumoniae. Epidemiol Infect 2011; 139: 1307-1316.

4 Bordon J, Peyrani P, Brock GN, et al. The presence of pneumococcal bacteremia does not influence clinical outcomes in patients with community-acquired pneumonia: results from the Community-Acquired Pneumonia Organization (CAPO) International Cohort study. Chest 2008; 133: 618-624.

5 Garnacho-Montero J, Garcia-Cabrera E, Diaz-Martin A, et al. Determinants of outcome in patients with bacteraemic pneumococcal pneumonia: importance of early adequate treatment. Scand J Infect Dis 2010; 42: $185-192$.

6 Mandell LA, Wunderink RG, Anzueto A, et al. Infectious Diseases Society of America/American Thoracic Society consensus guidelines on the management of community-acquired pneumonia in adults. Clin Infect Dis 2007; 44: Suppl. 2, S27-S72.

7 Woodhead M, Blasi F, Ewig S, et al. Guidelines for the management of adult lower respiratory tract infections--full version. Clin Microbiol Infect 2011; 17; Suppl. 6, E1-E59.

8 Metersky ML, Ma A, Bratzler DW, et al. Predicting bacteremia in patients with community-acquired pneumonia. Am J Respir Crit Care Med 2004; 169: 342-347.

9 Falguera M, Trujillano J, Caro S, et al. A prediction rule for estimating the risk of bacteremia in patients with community-acquired pneumonia. Clin Infect Dis 2009; 49: 409-416.

10 Fine MJ, Auble TE, Yealy DM, et al. A prediction rule to identify low-risk patients with community-acquired pneumonia. N Engl J Med 1997; 336: 243-250.

11 Lim WS, van der Eerden MM, Laing R, et al. Defining community acquired pneumonia severity on presentation to hospital: an international derivation and validation study. Thorax 2003; 58: 377-382.

12 Rice LB. Federal funding for the study of antimicrobial resistance in nosocomial pathogens: no ESKAPE. J Infect Dis 2008; 197: 1079-1081.

13 Torres A, Barberan J, Falguera M, et al. Guia multidisciplinar para la valoracion pronóstica, diagnostico y Tratamiento de la neumonia adquirida en la comunidad [Multidisciplinary guidelines for the management of community-acquired pneumonia]. Med Clin (Barc) 2013; 140: 223.e1-223.e19.

14 Hosmer D, Lemeshow S. Applied logistic regression. New York, John Wiley \& Sons Inc., 1989.

15 Waterer GW, Wunderink RG. The influence of the severity of community-acquired pneumonia on the usefulness of blood cultures. Respir Med 2001; 95: 78-82.

16 Riedel S, Bourbeau P, Swartz B, et al. Timing of specimen collection for blood cultures from febrile patients with bacteremia. J Clin Microbiol 2008; 46: 1381-1385.

17 Mylotte JM, Tayara A. Blood cultures: clinical aspects and controversies. Eur J Clin Microbiol Infect Dis 2000; 19: $157-163$.

18 Shorr AF, Zilberberg MD, Reichley R, et al. Validation of a clinical score for assessing the risk of resistant pathogens in patients with pneumonia presenting to the emergency department. Clin Infect Dis 2012; 54: 193-198.

19 Aliberti S, Di Pasquale M, Zanaboni AM, et al. Stratifying risk factors for multidrug-resistant pathogens in hospitalized patients coming from the community with pneumonia. Clin Infect Dis 2012; 54: 470-478.

20 Aliberti S, Cilloniz C, Chalmers JD, et al. Multidrug-resistant pathogens in hospitalised patients coming from the community with pneumonia: a European perspective. Thorax 2013; 68: 997-999. 